\title{
The mitochondrial phylogeny of land plants shows support for Setaphyta under composition-heterogeneous substitution models
}

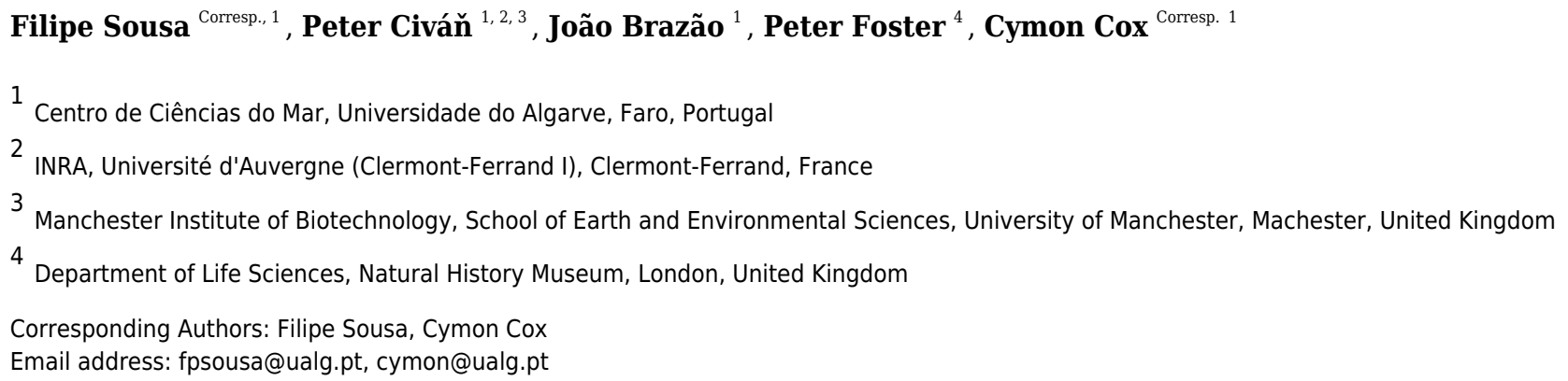

Congruence among analyses of plant genomic data partitions (nuclear, chloroplast and mitochondrial) is a strong indicator of accuracy in plant molecular phylogenetics. Recent analyses of both nuclear and chloroplast genome data of land plants (embryophytes) have, controversially, been shown to support monophyly of both bryophytes (mosses, liverworts, and hornworts) and tracheophytes (lycopods, ferns, and seed plants), with mosses and liverworts forming the clade Setaphyta. However, relationships inferred from mitochondria are incongruent with these results, and typically indicate paraphyly of bryophytes with liverworts alone resolved as the earliest-branching land plant group. Here, we reconstruct the mitochondrial land plant phylogeny from a newly compiled data set. When amonglineage composition heterogeneity is accounted for in analyses of codon-degenerate nucleotide and amino acid data, the clade Setaphyta is recovered with high support, and hornworts are supported as the earliest-branching lineage of land plants. These new mitochondrial analyses demonstrate partial congruence with current hypotheses based on nuclear and chloroplast genome data, and provide further incentive for revision of how plants arose on land. 


\section{The mitochondrial phylogeny of land plants shows}

2 support for Setaphyta under composition-

3 heterogeneous substitution models

4

5

8

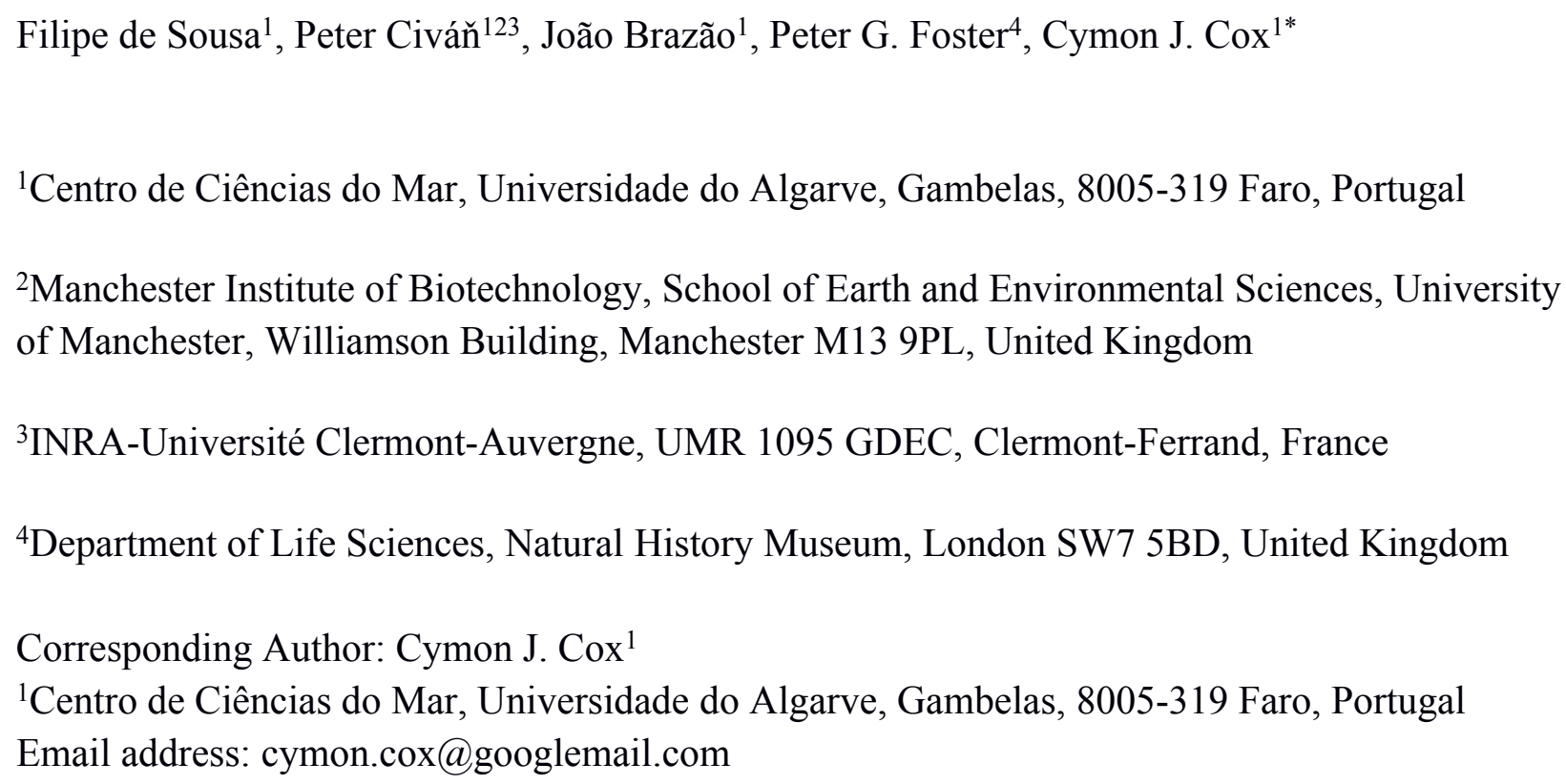

\section{Abstract}

Congruence among analyses of plant genomic data partitions (nuclear, chloroplast and mitochondrial) is a strong indicator of accuracy in plant molecular phylogenetics. Recent analyses of both nuclear and chloroplast genome data of land plants (embryophytes) have, controversially, been shown to support monophyly of both bryophytes (mosses, liverworts, and hornworts) and tracheophytes (lycopods, ferns, and seed plants), with mosses and liverworts forming the clade Setaphyta. However, relationships inferred from mitochondria are incongruent with these results, and typically indicate paraphyly of bryophytes with liverworts alone resolved as the earliest-branching land plant group. Here, we reconstruct the mitochondrial land plant phylogeny from a newly compiled data set. When among-lineage composition heterogeneity is accounted for in analyses of codon-degenerate nucleotide and amino acid data, the clade 
36 Setaphyta is recovered with high support, and hornworts are supported as the earliest-branching 37 lineage of land plants. These new mitochondrial analyses demonstrate partial congruence with current hypotheses based on nuclear and chloroplast genome data, and provide further incentive for revision of how plants arose on land.

\section{Introduction}

The embryophytes, or land plants, share a green algal ancestor (McCourt, Delwiche \& Karol 2004) that colonized terrestrial environments between 515.1- 470.0 Ma (Morris et al. 2018) and comprise gametophyte-dominant lineages, collectively known as bryophytes, and a sporophytedominant lineage, the tracheophytes. Establishing the phylogenetic relationships between bryophytes (mosses, liverworts and hornworts) and tracheophytes (a monophyletic lineage that includes lycopods, ferns and seed plants) is therefore fundamental to understanding the evolution of plants on land. However, phylogenetic inferences of relationships among embryophytes drawn from molecular data of the nuclear (Finet et al. 2010; Wodniok et al. 2011; Wickett et al. 2014), chloroplast (Cox et al. 2014; Ruhfel et al. 2014; Zhong et al. 2013; Bell et al. 2020), and mitochondrial (Turmel, Otis \& Lemieux 2013; Liu et al. 2014; Bell et al. 2020) genomes have long remained conflicting. These incongruences are likely due to molecular evolutionary processes that are especially apparent at deep timescales, such as multiple substitutions on the same site, that lead to loss of phylogenetic signal, and heterogeneity in substitution process patterns among sites and among lineages (Cox 2018). Phylogenetic patterns commonly observed among embryophytes included a sister-group relationship between hornworts and other embryophytes (Hedderson, Chapman \& Rootes 1996; Malek et al. 1996; Nishiyama and Kato 1999; Wickett et al. 2014), between liverworts and other embryophytes (Lewis, Mishler \& Vilgalys 1997; Karol et al. 2001; Qiu et al. 2006; Gao, Su \& Wang 2010; Karol et al. 2010; Clarke, Warnock \& Donoghue 2011), or between embryophytes and a clade uniting mosses and liverworts (Karol et al. 2010). An alternative pattern shows an initial split between the bryophytes and the tracheophytes (Hori, Lim \& Osawa 1985; Nishiyama et al. 2004; Goremykin and Hellwig 2005; Cox et al. 2014; Wickett et al. 2014; Puttick et al. 2018; Sousa et al. 2019; Leebens-Mack et al. 2019), implying the monophyly of both lineages. Nevertheless, the results are very much dependent on data and methodology, with authors typically presenting competing hypotheses. For instance, several recent phylogenomic analyses based on large nuclear data sets 
67 and extensive taxon sampling (e.g. Wickett et al. 2014; Leebens-Mack et al. 2019) have been

68 equivocal. These studies presented monophyletic-bryophyte phylogenies based on multi-species

69

70

71

72

73

74

75

76

77

78

79

80

81

82

83

84

85

86

87

88

89

90

91

92

93

94

95

96

coalescent supertrees, but concatenated analyses of the same data resulted in trees in which the bryophytes were paraphyletic. Consequently, the authors were unable to provide arguments for which hypothesis was to be preferred. Indeed, the efficacy and suitability of using multi-species coalescent summary analyses versus concatenated data analyses for phylogenies with deep timescales is currently a topic of considerable debate (e.g. Tonini et al. 2015; Edwards et al. 2016; Springer and Gatesy 2016). However, it should be noted that concatenated analyses of nuclear data do support a monophyletic bryophyte clade when modeling composition heterogeneity across the tree, although restricted analytical conditions, namely reduced taxon and site sampling, are currently necessary to decrease computational complexity when using treeheterogeneous composition models. For instance, to use these models, Sousa et al (2019) analysed a reduced amino acid data set of 26 taxa and 100 genes, while Puttick et al. (2018) analysed Dayhoff-recoded data, that reduces the amino acid data to only six character states. Nevertheless, these tree-heterogeneous composition models are demonstrably better-fitting and are therefore likely more accurate and reliable than the analyses of larger data sets that used simpler and poorer-fitting models (Cox 2018).

Most analyses of land plant relationships have been based on chloroplast data and have typically shown the bryophytes to be paraphyletic (see Table 1 in Cox 2018). More recent phylogenetic analyses using models that account for saturation and composition treeheterogeneity have, however, indicated that the bryophytes form a monophyletic group, and the authors provided arguments as to why these analyses using better-fitting models are to be preferred (Cox et al. 2014). In contrast, few land plant analyses of mitochondrial data have been presented, but all have indicated that the bryophytes form a paraphyletic group (i.e. Duff and Nickrent 1999; Groth-Malonek et al. 2004; Liu et al. 2014). The most recent and extensive phylogenetic analyses of plant mitochondrial genomes using tree-homogeneous composition models have shown that protein-coding nucleotide data place mosses as the sister-group to the remaining embryophytes, whereas amino acid data show a split between liverworts and the remaining embryophytes (Liu et al. 2014). However, this placement of liverworts as the sistergroup to the remaining embryophytes contradicts recent nuclear and chloroplast phylogenies 
97 which show high support for the clade Setaphyta, that groups liverworts with mosses (Cox et al. 98 2014; Puttick et al. 2018; Sousa et al. 2019).

Our confidence in any evolutionary hypotheses regarding land plant relationships would

100

101

102

103

104

105

106

107

108

109

110

111

112

113

114

115

116

117

118

119

120

121

122

123

124

125

126

127

increase greatly if phylogenetic inferences made from all three plant genomic compartments were not in conflict. In this study we investigate the mitochondrial phylogeny of land plants by applying better-fitting evolutionary models that account for composition tree-heterogeneity to a newly compiled data set of mitochondrial loci that includes sequences from three newly assembled genomes from the Coleochaetales and Zygnematales. We assemble a mitochondrial land plant data set of 26 taxa, which includes all major lineages of land plants and one of the putative most closely-related lineages to land plants, the Zygnematales. Assuming that both bryophytes and tracheophytes are likely monophyletic on the species tree (Sousa et al. 2019), the taxon sampling is deliberately restricted to include a proportional selection of bryophytes (11 taxa) and tracheophytes (10) as symmetrical trees improve estimation (Huang and Knowles 2009) and minimise long branch attraction (Philippe and Laurent 1998). Where possible, taxa were chosen to span what is currently considered the ancestral node of each bryophyte lineage (or the oldest ancestral node possible given the currently available data), thereby attempting to minimise the length of the sub-tending branch of each bryophyte clade and reduce longbranches. Most importantly, a smaller data set enables us to use better-fitting models that account for among-lineage and among-site composition heterogeneity that are computationally intractable for large data sets. This reduced sampling is in contrast to other studies which typically include many more taxa from highly derived lineages (especially angiosperms) whose inclusion, we maintain, has little impact on the resolution among major lineages (the question under consideration), but severely restricts the complexity of models that can be used and therefore the reliability of the inferences. Notably, a recent large-scale analysis of plant transcriptomes, despite the inclusion of 1155 taxa and 410 genes, was unable to resolve many of the long-standing contentious phylogenetic relationships, such as the relationships among the major lineages of land plants (Fig. 3 in Leebens-Mack et al. 2019). Indeed, rather than just including all available data injudiciously, and thereby restrict the complexity of models that can be applied for phylogenetic inference, it is important to consider which data should be included in a particular analysis: the choice of data should reflect carefully the question being addressed and its suitability for analysis by better-fitting models of molecular evolution.

Peer) reviewing PDF | (2019:12:43726:2:0:NEW 10 Mar 2020) 
Materials \& Methods

131

\section{Sampling of mitochondrial genomes}

133

134

135

136

137

138

139

140

141

142

143

144

145

146

147

148

149

150

151

152

153

154

155

156

157

158

We sampled 21 taxa representing the major lineages of land plants, plus 5 green algae species as outgroup taxa. The taxa sampled for this study were: Coleochaetales (Chaetosphaeridium globosum, Coleochaete scutata), Zygnematales (Closterium baillyanum, Gonatozygon brebissonii, Roya anglica), liverworts (Aneura pinguis. Marchantia polymorpha, Pleurozia purpurea, Treubia lacunosa), mosses (Atrichum angustatum, Bartramia pomiformis, Physcomitrella patens, Sphagnum palustre, Tetraphis pellucida), hornworts (Megaceros aenigmaticus, Phaeoceros laevis), lycophytes (Huperzia squarrosa, Isoetes engelmannii), pteridophytes (Ophioglossum californicum, Psilotum nudum), and spermatophytes (Brassica napus, Cycas taitungensis, Ginkgo biloba, Liriodendron tulipifera, Oryza sativa, Welwitschia mirabilis).

Algal cultures for Coleochaete scutata (SAG 3.91) and Gonatozygon brebissonii (SAG 292) were obtained from the Culture Collection of Algae (SAG) (Georg-August-Universität Göttingen, Germany), and the algal culture of Roya anglica (ACOI 799) was obtained from the Coimbra Collection of Algae (ACOI) (Universidade do Coimbra, Coimbra, Portugal). The mitochondrial genomes of the three taxa were sequenced and assembled de-novo using standard methods as described in (Civáň et al. 2014), and annotated with the aid of Mitofy (Alverson et al. 2010) and NCBI BLAST analyses (Altschul et al. 1990). The remainder of the genomes were retrieved from NCBI GenBank (Bethesda, USA). The list of species samples, their classification, and the source and accession numbers of the sequences used are shown in Table 1.

\section{Alignment and model testing}

The sequences of each of 43 mitochondrial protein-coding genes were aligned using the program MAFFT (vers. 6.8; Katoh and Toh 2008). Nucleotide alignments were manually edited in Geneious (vers. 9; http://www.geneious.com) to remove out-of-frame indels, misaligned portions, premature stop codons, positions with less than 50\% taxon occupancy, and ambiguities. Genes that were missing with the algal outgroup taxa (Coleochaetales and Zygnematales) or 
159 were under $200 \mathrm{bp}$ in length were discarded. The final data set consisted of 36 genes for 26 taxa, 160 with a missing gene occupancy of $9.5 \%$. Amino acid alignments were generated by translation 161 from each of the 36 nucleotide matrices using SeaView (vers. 4.5.4; Gouy, Guindon \& Gascuel 162 2009). The best-fitting substitution models for the 36 amino acid alignments were inferred in 163 PartitionFinder (Lanfear et al. 2012) using the BIC selection criterion. The stmtREV (Liu et al. 164 2014) with a gamma-distribution of among-site rates $(+G)$ model was the best-fitting model for 165 all genes but one, for which the chosen model was JTT+G (Jones, Taylor \& Thornton 1992). 166

167 168

169

170

171

172

173

174

175

176

177

178

179

180

181

182

183

184

185

186

187

188

189

\section{Gene tree estimation and monophyly test}

Gene trees were estimated from individual nucleotide matrices, using the general time-reversible model (Rodriguez et al. 1990) with a gamma rate distribution and estimated base frequencies $(\mathrm{GTR}+\mathrm{G}+\mathrm{Fest})$, and from amino acid matrices, using the best-fitting models inferred inPartitionFinder ( $\left.\operatorname{stmtREV}+\mathrm{G}+\mathrm{F}_{\text {est }}, \mathrm{JTT}+\mathrm{G}+\mathrm{F}_{\text {est }}\right)$. Bayesian MCMC analyses were performed with P4 (Foster 2004) using tree-homogeneous (henceforth referred to as CV1, i.e. one composition vector) and tree-heterogeneous (NDCH) composition models. Each analysis had two independent runs which were assessed for convergence by calculation of the marginal likelihood (chains were considered to have converged if they differed by $<10$ units) and the average standard-deviation of split support between trees sampled from the posterior distributions (chains were considered to have converged if $<0.01$ ). Posterior predictive simulations of the $\mathrm{X}^{2}$ test statistic of composition homogeneity was used to assess composition fit (Foster 2004). For each gene, 50\% majority-rule consensus trees were generated from the best-fitting analyses of the nucleotide and amino acid data.

Gene tree topologies inferred from each of the 36 amino acid alignments were tested using "gene genealogy interrogation" (GGI; Arcila et al. 2017) to ascertain whether the nonmonophyly of the five major lineages under scrutiny (hornworts, liverworts, mosses, tracheophytes, and the outgroups) was statistically supported by the data. We were to consider any gene that supported the non-monophyly of one of these clades as aberrant and not suitable for inclusion in the combined analyses, as the monophyly of hornworts, liverworts, mosses, tracheophytes and embryophytes has been consistently recovered in molecular phylogenies (e.g. Qiu et al. 2006; Liu et al. 2014). Optimal trees for each gene were compared to each of fifteen 
190 constraint trees representing all possible topologically resolved combinations of the five 191 monophyletic groups, using a nonparametric bootstrapping test. The results were assessed for 192 statistical significance with the Approximately Unbiased (AU) test (Shimodaira 2002) in 193 CONSEL (vers. 0.1k; Shimodaira and Hasegawa 2001). Optimal trees were estimated in 194 RAxML (MPI-compiled vers. 8.2.8; Stamatakis 2014) using the model stmtREV $+\mathrm{G}+\mathrm{F}_{\text {est }}$ and 195 starting from 20 random trees. Constraint trees were written in Newick format with internal 196 branches within each of the five major clades collapsed to a polytomy. Each constraint tree was 197 optimized in RAxML under the stmtREV $+\mathrm{G}+\mathrm{F}_{\text {est }}$ model. Constraint topologies were considered 198 statistically supported by the data if the p-value of the AU test was equal or greater than 0.05 , 199 meaning that the monophyly of each of the five lineages could not be rejected. In every gene, at 200 least one of the constraint topologies was supported by the data, meaning that the monophyly of 201 each clade could not be rejected, thus all 36 genes were included in downstream analyses.

202

203

204

205

206

207

208

209

210

211

212

213

214

215

216

217

218

219

220

\section{Analyses of concatenated nucleotide data}

A nucleotide alignment with 24,864 characters was obtained from the concatenation of the 36

individual genes. A second concatenated nucleotide matrix was constructed by codon-degenerate recoding of the data where ambiguity codes are used to negate synonymous substitutions (Criscuolo and Gribaldo 2010; Cox et al. 2014). Bayesian MCMC analyses were performed on the concatenated and codon-degenerate nucleotide matrices using a tree-homogeneous composition model (CV1; 2 replicates) and the tree-heterogeneous composition (NDCH2; 4 replicates) model, as implemented on P4. In contrast to the original NDCH model (Foster 2004) that allows an a priori defined number of compositions to evolve on the tree, the NDCH2 model estimates a separate composition for each node of the tree, constrained by a sampled Dirichlet prior on how much the composition vectors may differ from the empirical composition. Model fit to composition heterogeneity was inferred during the Bayesian MCMC with posterior predictive simulations of the $\mathrm{X}^{2}$ statistic of composition homogeneity, where $\mathrm{p}$-values equal or greater than 0.05 indicate acceptance of the model. The $\mathrm{GTR}+\mathrm{G}+\mathrm{F}_{\text {est }}$ model of substitution was used for for all MCMC of nucleotide and degenerate nucleotide data. Marginal likelihoods were estimated in P4 according to the Eqn 16 method of Newton and Raftery (1994).

\section{Analyses of concatenated amino acid data}


221 An amino acid alignment with 8,288 characters was obtained by concatenation of the amino acid 222 translations of the 36 genes. Bayesian MCMC analysis was performed on the concatenated 223 amino acid data using both tree-homogeneous composition ( $\mathrm{stmtREV}+\mathrm{G}+\mathrm{F}_{\mathrm{CV} 1}$; 2 replicates) and 224 tree-heterogeneous composition ( $\mathrm{stmtREV}+\mathrm{G}+\mathrm{F}_{\mathrm{NDCH} 2} ; 4$ replicates) models, with the fit of the 225 composition evaluated by posterior predictive simulations as described for the nucleotide data. In 226 addition, a Bayesian MCMC analysis was also performed using PhyloBayes (MPI-compiled 227 vers. 1.6; Lartillot, Lepage \& Blanquart 2009) under the model stmtREV+G+F

Alignments of individual genes, the concatenated data, and the resulting tree files of each 229 analysis are available on Zenodo (doi: 10.5281/zenodo.3554149). All ML analyses of the concatenated nucleotide and amino acid data sets were consistent with the homogeneous Bayesian MCMC analyses and are not reported here, but the resulting tree files are also available on Zenodo.

233

\section{Results}

Nucleotide and codon-degenerate data

All individual genes were best-fit by a tree-heterogeneous composition model with two composition vectors on the tree (CV2; Table S1). Majority-rule consensus trees resulting from the best-fitting Bayesian MCMC analyses of individual genes had low resolution in general, but liverworts were supported ( $>95 \%$ posterior probability (PP)) as the earliest-branching lineage in two genes (nad3 and nad5), whereas the mosses were supported as the earliest-branching lineage in one gene $(\mathrm{ccm} \mathrm{C})$. All other resolutions of the bryophyte lineages relative to the tracheophyte clade were not statistically supported, and the Setaphyta clade was not resolved in any gene tree. homogeneous composition (CV1), resulted in a tree with mosses as the sister-group to the remaining land plants $(\mathrm{PP}=1.0$; Figure 1a; Figure $\mathrm{S} 1)$, and hornworts as sister-group to the tracheophytes $(\mathrm{PP}=1.0)$. In contrast, the analysis of the degenerate data set under a homogeneous base composition (Figure 1c; Figure S2) returned a tree with liverworts the earliest-branching lineage of embryophytes $(\mathrm{PP}=1.0)$ and hornworts the sister-group to tracheophytes $(\mathrm{PP}=0.93)$. However, the homogeneous model was rejected for both data sets by the posterior predictive simulation of the $\mathrm{X}^{2}$ statistics of homogeneity, with a tail-area probability of 0.0 , thereby indicating that the data were not composition homogeneous. The tree- 
252 heterogeneous composition analysis (NDCH2) of the concatenated nucleotide data also resulted 253 in mosses supported as the earliest-diverging land plant lineage $(\mathrm{PP}=0.98$; Figure 1b; Figure $254 \mathrm{~S} 3)$, but placed liverworts as the sister-group to tracheophytes ( $\mathrm{PP}=0.94)$. The NDCH2 model

255

256

257

258

259

260

261

262

263

264

265

266

267

268

269

270

271

272

273

274

275

276

277

278

279

280

281

282 was a good fit to the data according to the posterior predictive simulation $\left(X^{2} p=0.99\right)$. When analysing the codon-degenerate data, the NDCH2 model recovered hornworts as the sister-group to the remaining land plants with full branch support ( $\mathrm{PP}=1.0$; Figure 1d; Figure S4), and mosses fully supported $(\mathrm{PP}=1.0)$ as the sister-group to liverworts (i.e. the clade Setaphyta). The $\mathrm{NDCH} 2$ model was not a good statistical fit to the data for the best scoring MCMC run, according to the $\mathrm{X}^{2}$ posterior predictive test $\left(\mathrm{X}^{2} \mathrm{p}=0.038\right.$; Figure $\left.\mathrm{S} 4\right)$.

\section{Amino acid data}

Individual mitochondrial protein alignments were best-fit by both homogeneous and heterogeneous composition models, with some being best-fit by a model with up to four compositions (CV4), indicating that they are highly heterogeneous in composition among lineages (Table S1). Majority-rule consensus trees of best-fitting Bayesian MCMC analyses of individual proteins were poorly supported regarding relationships among bryophyte lineages. Indeed, only one tree $(\mathrm{ccm} C)$ showed any statistically supported resolution and placed the mosses as the earliest-branching lineage of embyrophytes. Although not statistically supported, one amino-acid tree showed bryophytes as a monophyletic group (rps7), and the clade Setaphyta was present in three others (atp4, $r p l 2$, and $s d h 4)$.

When the concatenated amino acid data were analysed with a tree-homogeneous composition model (CV1), the resulting tree recovered liverworts as the sister-group to the remaining embryophytes without statistically significant support $(\mathrm{PP}=0.82)$, and hornworts as the sister-group to tracheophytes, also without support ( $\mathrm{PP}=0.82$; Figure 1e; Figure $\mathrm{S} 5)$. The homogeneous composition model did not fit the data $\left(\mathrm{X}^{2} \mathrm{p}=0.0\right)$. The Phylobayes stmtREV $+\mathrm{G}+\mathrm{F}_{\mathrm{CAT}}$ analysis (stationary, data-heterogeneous composition model) of the concatenated amino acid data resulted in an unsupported placement of liverworts as the sistergroup to the remaining embryophytes $(\mathrm{PP}=0.89$; Figure $\mathrm{S} 6)$. A posterior predictive composition homogeneity test using Phylobayes (readpb_mpi parameter-comp) showed that the data rejected the model and that the data were therefore composition tree-heterogeneous $(p=0.0)$. When the tree-heterogeneous composition NDCH2 model was used to model the concatenated protein data, 
283 the separate analyses did not converge on the same tree topology, although the NDCH2 model 284 was a good fit $\left(\mathrm{X}^{2} \mathrm{p}=0.1022\right)$. The tree obtained from the analyses with the best marginal 285 likelihood (- $\operatorname{lnLh} 142829.1129)$ supported hornworts as the earliest-branching lineage $(\mathrm{PP}=1.0)$, 286 with the liverworts as the sister-group to mosses ( $\mathrm{PP}=1.0$; Figs.1f, 2, S7).

287

\section{Discussion}

289

290

291

292

293

294

295

296

297

298

299

300

301

302

303

304

305

306

307

308

309

310

311

312

313

Gene tree discordance in mitochondrial data is likely due to mis-modeling and insufficient phylogenetic signal

Alternative hypothesis testing using nonparametric and parametric bootstrapping has been applied before to the mitochondrial land plant phylogeny to test the fit of the data (Liu et al. 2014). Here we chose a different approach, and used the optimized likelihood of constraint trees to identify genes that did not support the monophyly of the major embryophyte lineages (hornworts, liverworts, mosses, and tracheophytes) and of the outgroup. The optimal trees of the 36 mitochondrial genes, inferred under maximum-likelihood, show varied topologies, among which none is predominant. Because the four major land plant lineages are known to be monophyletic (as shown by many studies, e.g. Wickett et al. 2014) our concern was to identify gene trees that showed non-monophyly of one of these groups. The strategy we adopted allowed us to discern whether such topologies truly reflect underlying data or whether they are one among different topologies supported by the data. The AU test indicated, in all genes, that the monophyly of each land plant lineage was not statistically contradicted. This result suggests that the observed phylogenetic conflict among mitochondrial gene trees is unlikely to be explained by biological processes, such as horizontal gene transfer or duplication-loss, affecting specific lineages within each of the four major groups, and that any observed paraphyly of major groups on gene trees is probably the result of inadequate data modeling or paucity of phylogenetic signal.

Synonymous substitutions are responsible for the placement of mosses as the earliest-branching lineage of embryophytes

The tree inferred from the concatenated nucleotide data set of 36 mitochondrial genes shows mosses as the sister-group to the remaining land plants, as previous analyses of mitochondrial 
314 nucleotide data have shown (Liu et al. 2014). However, the mosses are replaced by the liverworts

315 in the same position when analysing codon-degenerate recoded data. Codon degenerate recoding

316 is used to eliminate synonymous substitutions, which are unconstrained by selection at the

317 protein level and therefore can be subject to high rates of substitution, and ultimately saturation

318 and loss of phylogenetic signal. Indeed, as the exclusion of synonymous substitutions is

319 sufficient to eliminate phylogenetic signal that supports mosses as the sister-group to the

320 remaining land plants, these results illustrate that despite being the slowest evolving genomic

321 compartment in plants, phylogenetic inferences from highly divergent mitochondrial genomes

322 are also affected by substitutional saturation due to the effect of high substitution rates.

323 Moreover, this observation implies that caution should be taken when invoking biological

324 explanations (e.g. hybridisation, incomplete lineage sorting) to explain incongruence among

325 phylogenies when inadequate modeling of the substitution process may represent a simpler

326 explanation.

327

328

329

Codon degenerate nucleotide data and protein data support Setaphyta under tree-heterogeneous models of substitution

330 With the nucleotide data, both the tree-homogeneous and NDCH2 tree-heterogeneous models

331 support mosses as the earliest-diverging group in the embryophytes. The likely incorrect

332 placement of mosses as the earliest-diverging group that is recovered with the best-fitting tree-

333 heterogeneous NDCH2 model suggests that homoplasy driven by high nucleotide substitution

334 rates (saturation) may overwhelm the ability of the model to correct for composition bias, hence

335 the need to use codon-degenerate recoded data in combination with tree-heterogeneous models.

336 Indeed, when codon-degenerate recoded data are used, contrasting supported relationships are

337 obtained under tree-homogeneous and tree-heterogeneous composition models. Whereas using a

338 homogeneous model for the analysis of the codon-degenerate data shows liverworts well

339 supported as the sister-group to other embryophytes, the tree-heterogeneous analysis (NDCH2)

340 model places liverworts as the sister-group to the mosses (clade Setaphyta), with maximum

341 support, and hornworts as the sister-group to all other embryophytes, also with maximum branch

342 support. These results demonstrate that the phylogenetic signal contained in non-synonymous

343 sites is also subject to composition biases and that tree-heterogeneous composition models are

344 required to model the data effectively. Contrasting results were also obtained when the amino 
345 acid data were analysed with tree-homogeneous and tree-heterogeneous models. The tree

346 inferred under the poorly-fitting $(\mathrm{P}=0.0)$ homogeneous model $(\mathrm{CV} 1)$ resolves liverworts as the

347 sister-group to the remaining land plants $(\mathrm{PP}=0.82)$. By contrast, the Bayesian $\mathrm{MCMC}$ run with

348 the highest marginal likelihood under the NDCH2 (fitting) model shows strong support for the

349 Setaphyta clade ( $\mathrm{PP}=1.0$ - mosses plus liverworts) with the hornworts as the earliest-branching

350 lineage of embryophytes $(\mathrm{PP}=1.0)$. Liu et al. (2014) observed topological congruence between

351 mitochondrial nucleotide and protein data that placed liverworts as the sister-group to all other

352 embryophytes, but this placement of liverworts received low branch support in different

353 analyses, and thus no firm conclusions regarding these cladogenic events were put foward.

354 Importantly, in that study no tree-heterogeneous analyses of the codon-degenerate data were

355 performed, nor was the protein data analysed with more than two composition vectors on the

356 tree. In contrast, our analyses of the codon-degenerate nucleotide data and amino acid data using

357 a better-fitting tree-heterogeneous model resulted in well supported, congruent topologies,

358 strengthening the argument in favour of the analyses presented here, that show support for the 359 clade Setaphyta.

360

361

The land plant mitochondrial phylogeny is partially congruent with nuclear and chloroplast 362 phylogenies

363 In contrast to previous analyses of the land plant mitochondrial phylogeny, we show that both 364 nucleotide and amino acid data carry signal that joins mosses and liverworts as sister lineages

365 (clade Setaphyta). This phylogenetic signal is typically obscured due to substitution saturation in

366 the nucleotide data and among-lineage composition bias in both the nucleotide and amino acid

367 data. In the nucleotide data, phylogenetic signal supporting mosses as the sister-group to the

368 remaining land plants is eliminated when codon-degenerate recoded data is analysed, and instead

369 the liverworts are found as the sister-group to all the remaining embryophytes under tree-

370 homogeneous composition models. However, it is only when a combination of codon-degenerate

371 recoding and a better-fitting tree-heterogeneous composition model is used that the mosses and

372 liverworts appear resolved as sister taxa, therefore suggesting that both substitutional saturation

373 and among-lineage composition heterogeneity are important evolutionary processes to be

374 modeled in the nucleotide data. Similarly, the unsupported placement of liverworts as the

375 earliest-branching lineage is obtained using tree-homogeneous composition models with the 
376 amino acid data, but better-fitting tree-heterogeneous composition models again support the 377 mosses plus liverwort clade.

$378 \quad$ Support for the moss-liverwort sister-group relationship has been found in trees

379 previously inferred from nuclear and chloroplast protein-coding data (e.g. Nishiyama et al. 2004;

380 Cox et al. 2014; Puttick et al. 2018; Sousa et al. 2019). The clade can be resolved by

381 mitochondrial data with our analyses, and therefore avoids the necessity of calling upon

382 biological explanations, such as hybridisation, to account for incongruence among the

383 phylogenies of the three plant genomes regarding the placement of mosses and liverworts.

384 However, if the placement of the hornworts as the earliest-branching lineage of embryophytes

385 does indeed reflect the true mitochondrial topology, then it is in conflict with the nuclear and

386 chloroplast data which suggest the bryophytes are likely monophyletic. A biological process

387 involving a rapid divergence of the hornworts from other bryophytes, after the tracheophyte-

388 bryophyte split, and the retention of a copy of the mitochondrion that was lost in all other

389 embryophyte lineages, could be invoked to explain the observed phylogenetic conflict. However,

390 the incongruence of the mitochondrial data could, of course, still be a result of mis-modeling or

391 lack of phylogenetic signal. It is likely that further sampling of mitochondrial genomes from

392 hornworts and other bryophyte lineages may aid resolution of the phylogeny, but such analyses

393 would only be informative if they were in combination with the heterogeneous composition

394 models that have been shown here to be necessary to correctly model the underlying processes of

395 mitochondrial evolution.

396

397

\section{Conclusions}

399

400 The main contribution of this study is the demonstration that liverworts are not the sister-group

401 to embryophytes in the land plant mitochondrial phylogeny, unlike earlier analyses of

402 mitochondrial genomes suggested (Liu et al. 2014). Instead, strong support is found for the clade

403 Setaphyta, corroborating support for this clade found in nuclear and plastid genomes, and

404 showing that the mitochondrial phylogeny of land plants is not strongly incongruent with the

405 nuclear and plastid phylogenies. Although the best-scoring tree found by analyses of amino acid

406 data places hornworts as sister-group to embryophytes, the monophyly of bryophytes, which is 
407 supported by evidence from nuclear and plastid genomes, cannot be strongly rejected.

408 Importantly, this study also shows that modeling of composition tree-heterogeneity in amino acid 409 data must not be disregarded, even in slower-evolving genomic regions such as plant 410 mitochondria.

411

412

413

414

415

416

417

418

419

420

421

422

423

424

425

426

427

428

429

430

431

432

433

434

435

\section{References}

Alverson AJ, Wei XX, Rice DW, Stern DB, Barry K, Palmer JD. 2010. Insights into the evolution of mitochondrial genome size from complete sequences of Citrullus lanatus and Cucurbita pepo (Cucurbitaceae). Molecular Biology and Evolution 27: 1436-1448.

Altschul SF, Gish W, Miller W, Myers EW, Lipman DJ. 1990. Basic local alignment search tool. Journal of Molecular Biology 215: 403-410.

Arcila D, Ortí G, Vari R, Armbruster JW, Stiassny ML, Ko KD, Sabaj MH, Lundberg J, Revell LJ, Betancur-R R. 2017. Genome-wide interrogation advances resolution of recalcitrant groups in the tree of life. Nature Ecology \& Evolution 1: 0020.

Bell, D., Lin, Q., Gerelle, W. K., Joya, S., Chang, Y., Taylor, Z. N.,Rothfels, C.J., Larsson, A., Villarreal, J.C., Li, F.-W., Pokorny, L., Szövényi, P., Crandall-Stotler, B., DeGironimo, L., Floyd, S.K., Beerling, D.J., Deyholos, M.K., von Konrat, M., Ellis, S., Shaw, A.J., Chen, T., Wong, G.K.-S., Stevenson, D.W., Palmer, J.D., Graham, S.W. (2020). Organellomic data sets confirm a cryptic consensus on (unrooted) land-plant relationships and provide new insights into bryophyte molecular evolution. American Journal of Botany 107: 91-115.

Civáň P, Foster PG, Embley MT, Seneca A, Cox CJ. 2014. Analyses of charophyte chloroplast genomes help characterize the ancestral chloroplast genome of land plants. Genome Biology and Evolution 6: 897-911.

Clarke JT, Warnock RCM, Donoghue PCJ. 2011. Establishing a time-scale for plant evolution. New Phytologist 192: 266-301. 
436 Cox CJ, Li B, Foster PG, Embley TM, Civáň P. 2014. Conflicting phylogenies for early land

437

438

439

440

441

442

443

444

445

446

447

448

449

450

451

452

453

454

455

456

457

458

459

460

461

462

463

464

465

466

plants are caused by composition biases among synonymous substitutions. Systematic Biology 63: 272-279.

Cox CJ. 2018. Land plant molecular phylogenetics: a review with comments on evaluating incongruence among phylogenies. Critical Reviews in Plant Sciences 37: 113-127.

Criscuolo A, Gribaldo S. 2010. BMGE (Block Mapping and Gathering with Entropy): a new software for selection of phylogenetic informative regions from multiple sequence alignments. BMC Evolutionary Biology 10: 210.

Duff RJ, Nickrent DL. 1999. Phylogenetic relationships of land plants using mitochondrial small subunit rDNA sequences. American Journal of Botany 86: 372-386.

Edwards SV, Xi Z, Janke A, Faircloth BC, McCormack JE, Glenn TC, Zhong B, Wu S, Lemmon EM, Lemmon AR, Leaché AD. 2016. Implementing and testing the multispecies coalescent model: a valuable paradigm for phylogenomics. Molecular Phylogenetics and Evolution 94: 447-462.

Finet C, Timme RE, Delwiche CF, Marlétaz F. 2010. Multigene phylogeny of the green lineage reveals the origin and diversification of land plants. Current Biology 20: 2217-2222.

Foster PG. 2004. Modeling compositional heterogeneity. Systematic Biology 53: 485-495.

Gao L, Su YJ, Wang T. 2010. Plastid genome sequencing, comparative genomics, and phylogenomics: Current status and prospects. Journal of Systematics and Evolution 48: 77-93.

Goremykin VV, Hellwig FH. 2005. Evidence for the most basal split in land plants dividing bryophyte and tracheophyte lineages. Plant Systematics and Evolution 254: 93-103.

Gouy M, Guindon S, Gascuel O. 2009. SeaView version 4: a multiplatform graphical user interface for sequence alignment and phylogenetic tree building. Molecular Biology and Evolution 27: 221-224.

Groth-Malonek M, Pruchner D, Grewe F, Knoop V. 2004. Ancestors of trans-splicing mitochondrial introns support serial sister group relationships of hornworts and mosses with vascular plants. Molecular Biology and Evolution 22: 117-125.

Hedderson TA, Chapman RL, Rootes, WL. 1996. Phylogenetic relationships of bryophytes inferred from nuclear-encoded rRNA gene sequences. Plant Systematics and Evolution 200: 213-224.

Peer) reviewing PDF | (2019:12:43726:2:0:NEW 10 Mar 2020) 
467

468

469

470

471

472

473

474

475

476

477

478

479

480

481

482

483

484

485

486

487

488

489

490

491

492

493

494

495

496

497

Hori H, Lim B-L, Osawa S. 1985. Evolution of green plants as deduced from 5S rRNA sequences. Proceedings of the National Academy of Sciences U. S. A. 82: 820-823.

Huang H, Knowles LL. 2009. What is the danger of the anomaly zone for empirical phylogenetics? Systematic Biology 58: 527-536.

Jones DT, Taylor WR, Thornton JM. 1992. The rapid generation of mutation data matrices from protein sequences. Bioinformatics 8: 275-282.

Karol KG, McCourt RM, Cimino MT, Delwiche CF. 2001. The closest living relatives of land plants. Science 294: 2351-2353.

Karol KG, Karol KG, Arumuganathan K, Boore JL, Duffy AM, Everett KD, Hall JD, Hansen SK, Kuehl JV, Mandoli DF, Mishler BD, Olmstead RG. 2010. Complete plastome sequences of Equisetum arvense and Isoetes flaccida: implications for phylogeny and plastid genome evolution of early land plant lineages. BMC Evolutionary Biology 10: 321.

Katoh K, Toh H. 2008. Recent developments in the MAFFT multiple sequence alignment program. Brief. Bioinformatics 9: 286-298.

Lanfear R, Calcott B, Ho SY, Guindon S. 2012. PartitionFinder: combined selection of partitioning schemes and substitution models for phylogenetic analyses. Molecular Biology and Evolution 29: 1695-1701.

Lartillot N, Lepage T, Blanquart S. 2009. PhyloBayes 3: a Bayesian software package for phylogenetic reconstruction and molecular dating. Bioinformatics 25: 2286-2288.

Leebens-Mack J, Barker M, Carpenter E, Deyholos M, Gitzendanner M, Graham S, Grosse I, Li Z, Melkonian M, Mirarab S, Porsch M, Quint M, Rensing S, Soltis D, Soltis P, Stevenson D, Ullrich K, Wickett N, DeGironimo L, Edger P, Jordon-Thaden I, Joya S, Liu T, Melkonian B, Miles N, Pokorny L, Quigley C, Thomas P, Villarreal J, Augustin M, Barrett M, Baucom R, Beerling D, Benstein R, Biffin E, Brockington S, Burge D, Burris J, Burris K, Burtet-Sarramegna V, Caicedo A, Cannon S, Cebi Z, Chang Y, Chater C, Cheeseman J,Chen T, Clarke N, Clayton H, Covshoff S, Crandall-Stotler B, Cross H, DePamphilis C, Der J, Determann R, Dickson R, Di Stilio V, Ellis S, Fast E, Feja N, Field K, Filatov D, Finnegan P, Floyd S, Fogliani B, Garcia N, Gateble G, Godden G, Goh Q.-Y, Greiner S, Harkess A,Heaney J, Helliwell K, Heyduk K, Hibberd J, Hodel R, Hollingsworth P, Johnson M, Jost R,Joyce B, Kapralov M, Kazamia E, Kellogg E, Koch M, Von Konrat M, Konyves K, Kutchan T, Lam V, Larsson A, Leitch A, Lentz R, Li F.-W, Lowe A, Ludwig 
498

499

500

501

502

503

504

505

506

507

508

509

510

511

512

513

514

515

516

517

518

519

520

521

522

523

524

525

526

527

528

M, Manos P, Mavrodiev E, McCormick M, McKain M, McLellan T, McNeal J, Miller R, Nelson M, Peng Y, Ralph P,Real D, Riggins C, Ruhsam M, Sage R, Sakai A, Scascitella M, Schilling E, Schlosser E.-M, Sederoff H, Servick S, Sessa E, Shaw A, Shaw S, Sigel E, Skema C, Smith A, Smithson A, Stewart C, Stinchcombe J, Szovenyi P, Tate J, Tiebel H, Trapnell D, Villegente M, Wang C.-N, Weller S, Wenzel M, Weststrand S, Westwood J, Whigham D, Wu S, Wulff A, Yang Y, Zhu D, Zhuang C, Zuidof J, Chase M, Pires J, Rothfels C, Yu J, Chen C, Chen L, Cheng S, Li J, Li R, Li X, Lu H, Ou Y, Sun X, Tan X, Tang J, Tian Z, Wang F, Wang J, Wei X, Xu X, Yan Z, Yang F, Zhong X, Zhou F, Zhu Y, Zhang Y, Ayyampalayam S, Barkman T, Nguyen N.-P, Matasci N, Nelson D, Sayyari E, Wafula E, Walls R, Warnow T, An H, Arrigo N, Baniaga A, Galuska S, Jorgensen S, Kidder T, Kong H, Lu-Irving P, Marx H, Qi X, Reardon C, Sutherland B, Tiley G, Welles S, Yu R, Zhan S, Gramzow L, Theissen G, Wong G. K.-S. 2019. One thousand plant transcriptomes and the phylogenomics of green plants. Nature 574: 679-685.

Lewis LA, Mishler BD, Vilgalys R. 1997. Phylogenetic relationships of the liverworts (Hepaticae), a basal embryophyte lineage, inferred from nucleotide sequence data of the chloroplast gene rbcL. Molecular Phylogenetics and Evolution 7: 377-393

Liu Y, Cox CJ, Wang W, Goffinet B. 2014. Mitochondrial phylogenomics of early land plants: Mitigating the effects of saturation, compositional heterogeneity, and codon-usage bias. Systematic Biology 63: 862-878.

Malek O, Lättig K, Hiesel R, Brennicke A, Knoop V. 1996. RNA editing in bryophytes and a molecular phylogeny of land plants. The EMBO Journal 15: 1403-1411.

McCourt RM, Delwiche CF, Karol KG. 2004. Charophyte algae and land plant origins. Trends in Ecology \& Evolution 19: 661-666.

Morris JL, Morris JL, Puttick MN, Clark JW, Edwards D, Kenrick P, Pressel S, Wellman CH, Yang Z, Schneider H, Donoghue PC. 2018. The timescale of early land plant evolution. Proceedings of the National Academy of Sciences U. S. A. 115: E2274-E2283.

Newton MA, Raftery AE. 1994. Approximate Bayesian inference with the weighted likelihood bootstrap. Journal of the Royal Statistical Society B 56: 3-48.

Nishiyama T, Nishiyama T, Wolf PG, Kugita M, Sinclair RB, Sugita M, Sugiura C, Wakasugi T, Yamada K, Yoshinaga K, Yamaguchi K, Ueda K. 2004. Chloroplast phylogeny indicates that bryophytes are monophyletic. Molecular Biology and Evolution 21: 1813-1819. 
529 Nishiyama T, Kato M. 1999. Molecular phylogenetic analysis among bryophytes and

530

531

532

533

534

535

536

537

538

539

540

541

542

543

544

545

546

547

548

549

550

551

552

553

554

555

556

557

558

559 tracheophytes based on combined data of plasmid coded genes and the 18S rRNA gene. Molecular Biology and Evolution 16: 1027-1036.

Philippe H, Laurent J. 1998. How good are deep phylogenetic trees? Current Opinion in Genetics and Development 8: 616-623.

Puttick MN, Morris JL, Williams TA, Cox CJ, Edwards D, Kenrick P, Pressel S, Wellman CH, Schneider H, Pisani D, Donoghue PC. 2018. The interrelationships of land plants and the nature of the ancestral embryophyte. Current Biology 28: 733-745.

Qiu Y-L, Li L, Wang B, Chen Z, Knoop V, Groth-Malonek M, Dombrovska O, Lee J, Kent L, Rest J, Estabrook GF. 2006. The deepest divergences in land plants inferred from phylogenomic evidence. Proceedings of the National Academy of Sciences U. S. A. 103: 15511-15516.

Rodriguez F, Oliver JL, Marin A, Medina JR. 1990. The general stochastic model of nucleotide substitution. Journal of Theoretical Biology 142: 485-501.

Ruhfel BR, Gitzendanner MA, Soltis PS, Soltis DE, Burleigh JG. 2014. From algae to angiosperms--inferring the phylogeny of green plants (Viridiplantae) from 360 plastid genomes. BMC Evolutionary Biology 14: 23.

Shimodaira H. 2002. An approximately unbiased test of phylogenetic tree selection. Systematic Biology 51: 492-508.

Shimodaira H, Hasegawa M. 2001. CONSEL: for assessing the confidence of phylogenetic tree selection. Bioinformatics 17:1246-1247.

Sousa F, Foster PG, Donoghue PC, Schneider H, Cox CJ. 2019. Nuclear protein phylogenies support the monophyly of the three bryophyte groups (Bryophyta Schimp.). New Phytologist 222: 565-575.

Springer MS, Gatesy J. 2016. The gene tree delusion. Molecular Phylogenetics and Evolution 94, 1-33.

Stamatakis A. 2014. RAxML version 8: a tool for phylogenetic analysis and post-analysis of large phylogenies. Bioinformatics 30: 1312-1313.

Tonini J, Moore A, Stern D, Shcheglovitova M, Ortí G. 2015. Concatenation and species tree methods exhibit statistically indistinguishable accuracy under a range of simulated conditions. PLoS currents, 7. 
560 Turmel M, Otis C, Lemieux C. 2013. Tracing the evolution of streptophyte algae and their 561 mitochondrial genome. Genome Biology and Evolution 5: 1817-1835.

562 Wickett NJ, Mirarab S, Nguyen N, Warnow T, Carpenter C, Matasci N, Ayyampalayam S,

563

564

565

566

567

568

569

570

571

572

573

574

575

576 Barker MS, Burleigh JG, Gitzendanner MA, Ruhfel BR, Wafula E, Der JP, Graham SW, Mathews S, Melkonian M, Soltis DE, Soltis PS, Miles NW, Rothfels C-J, Pokorny L, Shaw AJ, DeGironimo L, Stevenson DW, Surek B, Villarreal JC, Roure B, Philippe H, dePamphilis CW, Chen T, Deyholos MK, Baucom RS, Kutchan TM, Augustin MM, Wang J, Zhang Y, Tian Z, Yan Z, Wu X, Sun X, Wong GK-S, Leebens-Mack J. 2014. Phylotranscriptomic analysis of the origin and early diversification of land plants. Proceedings of the National Academy of Sciences U. S. A. 111: E4859-E4868.

Wodniok S, Brinkmann H, Glöckner G, Heidel AJ, Philippe H, Melkonian M. Becker B. 2011. Origin of land plants: do conjugating green algae hold the key? BMC Evolutionary Biology 11: 104 .

Zhong B, Xi Z, Goremykin VV, Fong R, Mclenachan PA, Novis PM, Davis CC, Penny D. 2013. Streptophyte algae and the origin of land plants revisited using heterogeneous models with three new algal chloroplast genomes. Molecular Biology and Evolution 31: 177-183.

PeerJ reviewing PDF | (2019:12:43726:2:0:NEW 10 Mar 2020) 


\section{Table 1 (on next page)}

Accession table of the 26 samples used in this study.

For each species, the corresponding taxonomic group and NCBI GenBank accession numbers are shown. Accessions marked with an asterisk (*) correspond to newly assembled genomes. 


\begin{tabular}{|c|c|c|}
\hline species & taxonomic group & Accession \\
\hline Closterium baillyanum & Zygnematales & NC_022860 \\
\hline Gonatozygon brebissonii & Zygnematales & MK720950 * \\
\hline Roya anglica & Zygnematales & MK720948* \\
\hline Chaetosphaeridium globosum & Coleochaetales & NC_004118 \\
\hline Coleochaete scutata & Coleochaetales & MK720949* \\
\hline Megaceros aenigmaticus & hornworts & NC_012651 \\
\hline Phaeoceros laevis & hornworts & NC_013765 \\
\hline Aneura pinguis & liverworts & NC_026901 \\
\hline Marchantia polymorpha & liverworts & NC_001660 \\
\hline Pleurozia purpurea & liverworts & NC_013444 \\
\hline Treubia lacunosa & liverworts & NC_016122 \\
\hline Atrichum angustatum & mosses & NC_024520 \\
\hline Bartramia pomiformis & mosses & NC_024519 \\
\hline Physcomitrella patens & mosses & NC_007945 \\
\hline Sphagnum palustre & mosses & NC_024521 \\
\hline Tetraphis pellucida & mosses & NC_024290 \\
\hline Welwitschia_mirabilis & seed plants & NC_029130 \\
\hline Brassica napus & seed plants & NC_008285 \\
\hline Liriodendron tulipifera & seed plants & NC_021152 \\
\hline Oryza_sativa & seed plants & NC_011033 \\
\hline Cycas taitungensis & seed plants & NC_010303 \\
\hline Ginkgo biloba & seed plants & NC_027976 \\
\hline
\end{tabular}




\begin{tabular}{|l|l|l|}
\hline Ophioglossum californicum & ferns & NC_030900 \\
\hline Psilotum nudum & ferns & NC_030952, KX171639 \\
\hline Huperzia squarrosa & lycophytes & NC_017755 \\
\hline Isoetes engelmannii & lycophytes & FJ010859, FJ176330, FJ390841, \\
\hline
\end{tabular}

2 


\section{Figure 1}

A schematic representation of the topologies obtained from tree-homogeneous and tree-heterogeneous analyses of nucleotide, codon-degenerate nucleotide, and amino acid translation data.

Analyses of nucleotide data place mosses as the earliest-branching lineage of the embryophytes (1a, 1b). Analyses of codon-degenerate and amino acid data under treehomogeneous models place liverworts as the sister-group to the remaining embryophytes (1c, 1e), whereas analyses under tree-heterogeneous models show support for the clade Setaphyta (1d, 1f).

\section{data}

nucleotide
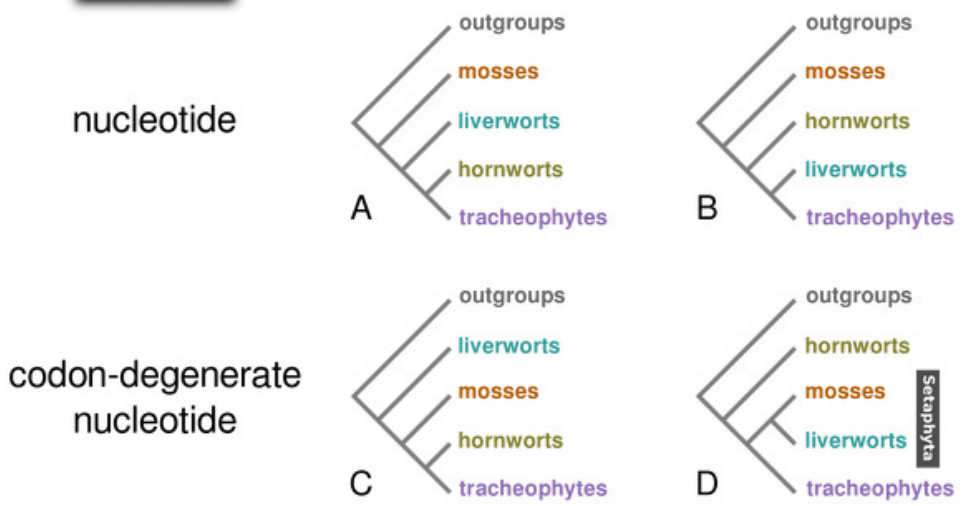

amino acid
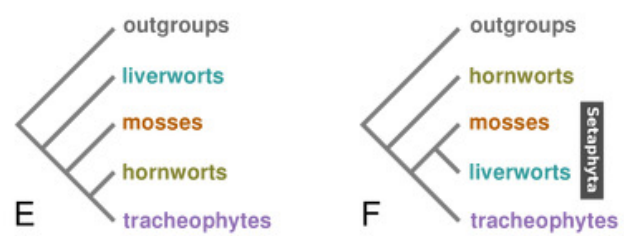

\section{model}

tree-homogeneous composition

tree-heterogeneous composition 


\section{Figure 2}

Majority-rule consensus tree inferred from the 36 gene, 26 taxon concatenated amino acid data.

Bayesian MCMC with a tree-heterogeneous composition model NDCH2, marginal likelihood $L_{h}=142829.112$. Additional analysis statistics can be found in the legend of Fig. S7. All branches fully supported ( $P P=1.0)$. Taxa indicated as follows: outgroups, grey; hornworts, olive green; liverworts, cyan blue; mosses, orange; vascular plants, violet. 


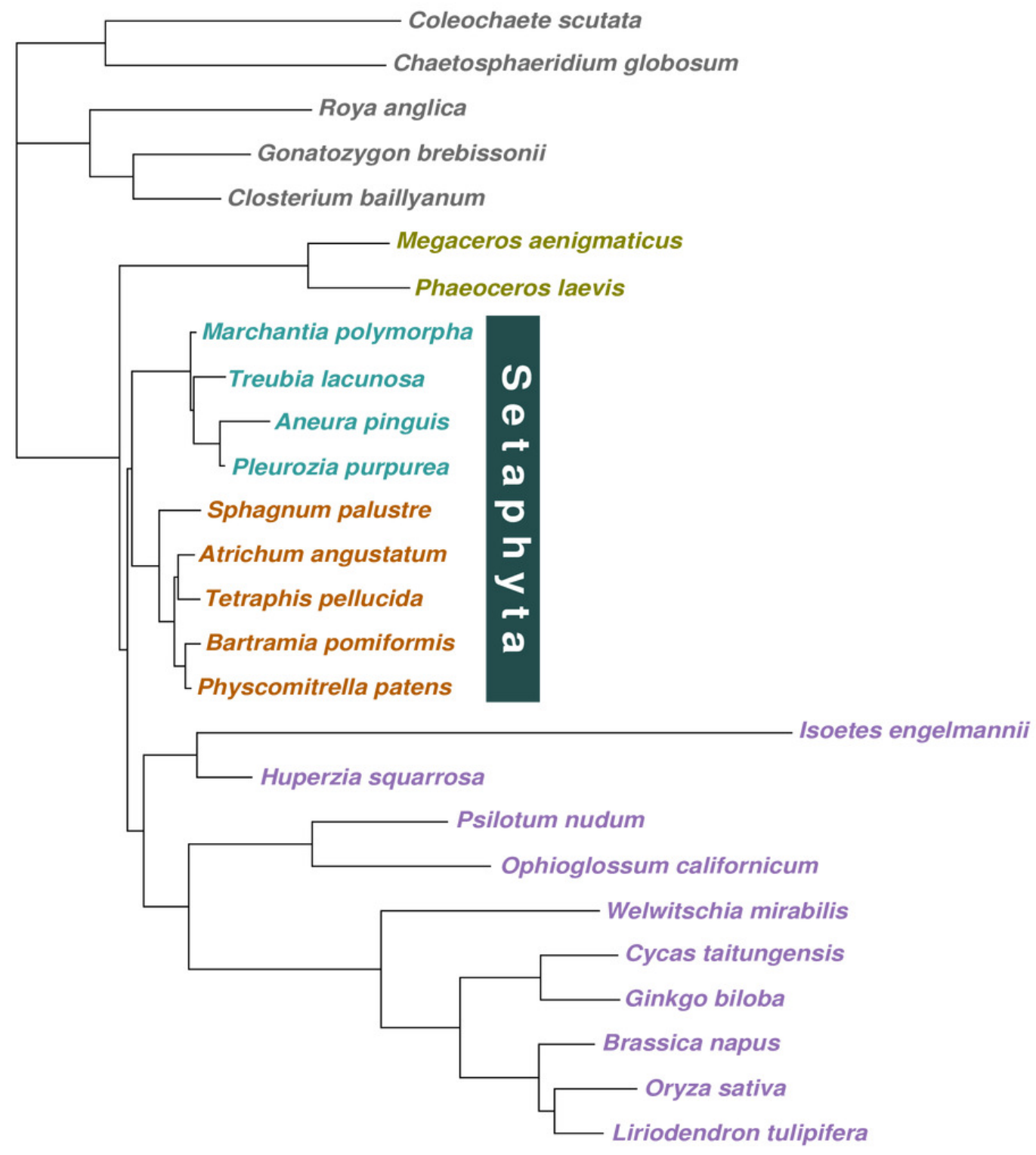

